\title{
High-Q Micro/Nanoresonators for Nonlinear/Quantum Photonics and Sensing
}

\author{
Qiang Lin \\ Department of Electrical and Computer Engineering, University of Rochester \\ Rochester, NY 14627, USA \\ qiang.lin@rochester.edu
}

\begin{abstract}
In this talk, we will discuss our recent progress in developing high-quality micro/nanoresonators on material platforms such as silicon, silicon carbide, and lithium niobate, whose excellent material properties exhibit great potential for broad photonic applications. We will focus on our recent efforts in applying them for nonlinear and quantum photonics, and for sensing applications.
\end{abstract}

\section{Keywords}

High-Q micro/nanoresonators; silicon carbide; lithium niobate; nonlinear photonics; quantum photonics; sensing.

\section{SUMMARY}

High-Q optical micro-/nano-resonators, with an exceptional capability of dramatically enhancing optical fields inside a small volume, are critical foundations for many areas of optical science, such as integrated nonlinear optics [1,2], cavity quantum electrodynamics $[3,4]$, cavity optomechanics [5], and biomedical sensing $[6,7]$. In general, the underlying materials play a crucial role since most applications rely on the linear/nonlinear optical property $[1,2]$, the mechanical property [5], the quantum confinement effect [3,4], defect characteristics [8], or thermal stability for proper operation $[6,7]$. In recent years, significant interest has been focused on developing micro-/nano-resonators on various material platforms, including dielectrics, semiconductors, and polymers.

Silicon carbide $(\mathrm{SiC})$ is a semiconductor $[9,10]$ with a wide band gap $(2.3-3.4 \mathrm{eV})$, a large refractive index $(\sim 2.6)$, extremely large Young's modulus $(\sim 450 \mathrm{GPa})$, exceptionally low mechanical damping, a significant thermal conductivitiy $(\sim 490 \mathrm{~W} /(\mathrm{m} \cdot \mathrm{K}))$, very high hardness and chemical inertness, and unique point defect characteristics [11]. Moreover, $\mathrm{SiC}$ exhibit many crystal forms with various material isotropy and anisotropy. Therefore, $\mathrm{SiC}$ is outstanding material for broad quantum photonic and optomechanical

Permission to make digital or hard copies of all or part of this work for personal or classroom use is granted without fee provided that copies are not made or distributed for profit or commercial advantage and that copies bear this notice and the full citation on the first page. Copyrights for components of this work owned by others than ACM must be honored. Abstracting with credit is permitted. To copy otherwise, or republish, to post on servers or to redistribute to lists, requires prior specific permission and/or a fee. Request permissions from permissions@ acm.org.

NANOCOM'16, September 28-30, 2016, New York, NY, USA

(C) 2016 ACM. ISBN 978-1-4503-4061-8/16/09.. $\$ 15.00$

DOI: http://dx.dei.org/10.1145/2967446.2967468 applications, particularly for sensing applications in harsh environment.

Lithium niobate $\left(\mathrm{LiNbO}_{3}\right)$, instead, exhibits exceptional nonlinear optical, electro-optical, piezoelectric, photoelastic, and ferroelectric properties $[12,13]$, with great potential as a universal material platform for making hybrid nanophotonic circuits for high-speed electro-optic and all optical signal processing, nonlinear wave generation, and photonic quantum state manipulation.

Their great potential for micro/nanophotonics has attracted significant interest in the past few years [14-24]. Unfortunately, the device development along these lines faces significant challenge due to the difficult in fabricating high-quality chip-scale photonic devices. We recently devoted considerable efforts in developing high-quality micro/nanophotonic devices in these material platforms. These devices allow us to observe intriguing nonlinear dynamics in thermal, optical, and mechanical degrees of freedom. Moreover, we have applied these devices for novel functionalities in nonlinear photonics, photonic signal processing, optomechanical sensing, and photonic quantum states generation. In this talk, we will discuss our recent progress along these lines.

\section{ACKNOWLEDGMENTS}

This work is supported in part by the DARPA SCOUT program through grant number W31P4Q-15-1-0007 from AMRDEC, and by NSF under grants numbers ECCS-1351697 and ECCS-1408517.

\section{REFERENCES}

[1] K. J. Vahala, "Optical microcavities," Nature 424, 839 (2003).

[2] T. J. Kippenberg, R. Holzwarth, and S. A. Diddams, "Microresonator-based optical frequency combs," Science 332, 555 (2011).

[3] [5] K. Kennessy, A. Badolato, M. Winger, D. Gerace, M. Atature, S. Gulde, S. Falt, E. L. Hu, and A. Imamoglu, "Quantum nature of a sgrongly coupled single quantum dot-cavity system," Nature 445, 896 (2007).

[4] S. Noda, M. Fujita, and T. Asano, "Spontaneous-emission control by photonic crystals and nanocavities," Nature Photon. 1, 449 (2007).

[5] M. Aspelmeyer, T. J. Kippenberg, and F. Marquardt, "Cavity Optomechanics," Rev. Mod. Phys. 86, 1391 (2014).

[6] X. Fan, I. M. White, S. I. Shopova, H. Zhu, J. D. Suter, and Y. Sun, "Sensitive optical biosensors for 
unlabeled targets: A review", Ana. Chem. Acta 620, 8 (2008).

[7] M. R. Foreman, J. d. Swaim, and F. Vollmer, "Whispering gallery mode sendors," Adv. Opt. Photon. 7, 168 (2015).

[8] A. Faraon, P. E. Barclay, C. Santori, K.-M. C. Fu, and R. G. Beausoleil, "Resonant enhancement of the zero-phonon emission from a colour centre in a diamond cavity," Nature. Photon. 5, 301 (2011).

[9] G. L. Harris, Properties of Silicon Carbide. (INSPEC, 1995).

[10] R. Maboudian, C. Carraro, D. G. Senesky, and C. S. Roper, "Advances in silicon carbide science and technology at the micro- and nanoscales," J. Vac. Sci. Technol. A 31, 050805 (2013).

[11] W. F. Koehl, et al, "Room temperature coherent control of defect spin qubits in silicon carbide," Nature 479, 85 (2011).

[12] R. S. Weis and T. K. Gaylord, "Lithium niobate: summary of physical properties and crystal structure," Appl. Phys. A 37, 191 (1985).

[13] K. K. Wong, Ed., Properties of lithium niobate," (IEE, INSPEC, 2002).

[14] B.-S. Song, S. Yamada, T. Asano, and S. Noda, "Demonstration of two-dimensional photonic crystals based on silicon carbide," Opt. Express 19, 11084 (2011).

[15] X. Lu, J. Y. Lee, P. X.L. Feng, and Q. Lin, "Silicon carbide microdisk resonator," Opt. Lett. 38, 1304 (2013).

[16] J. Cardenas, et al, "High Q SiC microresonators," Opt. Express 21, 16882 (2013).
[17] M. Radulaski, et al, "Photonic crystal cavities in cubic (3C) polytype silicon carbide films," Opt. Express 21, 32623 (2013).

[18] G. Calusine, A. Politi, and D. Awschalom, "Silicon carbide photonic crystal cavities with integrated color centers," Appl. Phys. Lett. 105, 011123 (2014).

[19] A. P. Magyar, et al, "High quality SiC microdisk resonators fabricated from monolithic epilayer wafers," Appl. Phys. Lett. 104, 051109 (2014).

[20] T. Wang, J. He. C. Lee, and H. Niu, "High-quality LiNbO3 microdisk resonators by undercut etching and surface tension reshaping," Opt. Express 20, 28119 (2012).

[21] C. Wang, M. J. Burek, Z. Lin, H. A. Atikian, V. Venkataraman, I. Huang, P. Stark, and M. Loncar, "Integrated high quality factor lithium niobate microdisk resonators," Opt. Express 22, 30924 (2014).

[22] J. Lin, Y. Xu, Z. Fang, M. Wang, J. Song, N. Wang, L. Qiao, W. Fang, and Y. Cheng, "Fabrication of high-Q lithium niobate microresonators using femtosecond laser micromaching," Sci. Rep. 5, 8072 (2015).

[23] R. Geiss, S. Saravi, A. Sergeyev, S. Diziain, F. Setzpfandt, F. Schrempel, R. Grange, E. Kley, A. Tunnermann, and T. Pertsch, "Fabrication of nanoscale lithium niobate waveguides for second-harmonic generation," Opt. Lett. 40, 2715 (2015).

[24] J. Wang, F. Bo, S. Wan, W. Li, F. Gao, J. Li, G. Zhang, and J. Xu, "High-Q lithium niobate microdisk resonators on a chip for efficient electro-optic modulation," Opt. Express 23, 23072 (2015). 